\title{
Socially constructed and structurally conditioned conflicts in territories of medical uncertainty
}

\author{
Olaug S. Lian ${ }^{1}$ Catherine Robson ${ }^{1}$
}

Published online: 27 November 2018

(C) The Author(s) 2018

\begin{abstract}
In territories of medical uncertainty, clinical encounters are highly contentious. To uncover maintaining mechanisms behind persistent conflicts, we explore the interactional dynamics of clinical encounters fused with medical uncertainty. Based on a thematic qualitative analysis of experiential texts from 385 people living with medically unexplained physical symptoms in Norway, UK, Ireland, USA and Canada, we explore patients' main expectations, how these expectations are met, and how their expectations and experiences are socially constructed and structurally conditioned. Five fundamental expectations are identified: Health professionals ought to (1) acknowledge the lack of medical knowledge, and be frank, open and curious about it; (2) believe patient experiences and accept their condition as "real"; (3) avoid blaming patients for their ailment; (4) demonstrate compassion, understanding and respect; and (5) share decision-making power with patients. Our participants experience unfulfilled expectations in all five areas. Both experiences and unfulfilled expectations are influenced by structural factors transpiring from the modern Western biomedical paradigm, and from cultural norms and values of its surrounding society. Structural and cultural forces obstruct team-oriented collaboration based on congruent understandings, mutual trust and reciprocated respect. Without such contextualisation, the interactional dynamics between patients and health professionals in clinical consultations cannot be exposed.
\end{abstract}

Keywords Medical uncertainty $\cdot$ Medically unexplained illness $\cdot$ Patient experiences $\cdot$ Clinical consultations - interaction $\cdot$ Non-epileptic seizures $\cdot$ Chronic fatigue/myalgic encephalomyelitis

Olaug S. Lian

olaug.lian@uit.no

1 Department of Community Medicine, Faculty of Health Sciences, University of Troms $\varnothing$ - The Arctic University of Norway, 9037 Troms $\varnothing$, Norway 


\section{Introduction}

As a social institution, the medical system is embedded in a continually changing sociocultural context. Together with medical and technological developments, the sociocultural context influences the organised framework and the informal culture of the medical system. Structural and cultural changes influence the roles of all actors involved, as well as the interactions between them (Parsons 1951; Freidson 1970).

In modern Western societies, the medical system (in which doctors are the main actors) is based on scientific knowledge that ought to be experimentally tested and proven, theoretically explained and technologically validated (Jewson 2009). The foundation for this paradigm includes the invention of the microscope in 1590, the development of bacteriology in the nineteenth century, and knowledge about the human anatomy and physiology developed during the last century. New knowledge and new technology has altered our understanding of health and illness, including our knowledge acquisition. Today, concepts and theories from biology, physics and chemistry are centrally placed in conceptions and explanations of diseases (Toombs 1992). The biomedical conceptualisation gives ontological primacy to the biological body, and human ailments are verified through technological tests. This perspective is often defined as the authoritative "truth", and expert accounts of health and illness are privileged over lay accounts. Given this cultural hierarchy of knowledge, it might be considered legitimate for health professionals - in the name of professional expertise- to remove from laymen the right to define the situation (Freidson 1970). The very foundation for this legitimacy lies in biomedical knowledge. When such knowledge is lacking, clinical encounters between doctors and patients become riddled by medical uncertainty. Far-reaching expansions of the medical jurisdiction, even beyond areas substantiated by medical knowledge (Busfield 2017), make this an increasingly relevant field of research.

Medical uncertainty involves a lack of certainty relating to diagnosis, prognosis, casual factors (aetiology) and/or likely results of various treatment options (Han et al. 2011). Most clinical encounters are to some extent infused with medical uncertainty in one way or another, for various reasons (Han et al. 2011). In this paper, we limit our discussion to situations in which the uncertainty originates from a lack of medical knowledge, more specifically the so-called medically unexplained physical symptoms. Our main reasons for choosing this focus are twofold. The first one is mainly theoretical: medically unexplained bodily ailments are theoretically and sociologically interesting because the ways in which we think about these phenomena reflects "complex social and political forces" (De Maio 2010, p. 2). Cultural norms and values affect the ways in which both lay and medical perceptions of these ailments are shaped, sustained and changed (Freidson 1970; Wright and Treacher 1982). The less medical knowledge we have, the larger the space for cultural imprints becomes. If we want to uncover the cultural contingency of medical constructions of health and illness, medically unexplained physical symptoms (MUPS) are particularly suitable. Another 
reason relates to clinical relevance: somatic symptoms with no detectable organic pathology are challenging for doctor-patient interactions because such conditions defy medical identification, medical explanation and evidence-based treatment. Conflictual debates about these conditions are expressed in medical journals (Lian and Bondevik 2015), in public media (de Wolfe 2009), on the internet (Lian and Nettleton 2015) and in consultation rooms (Banks and Prior 2001). MUPS-consultations are characterised as a war-zone (Lian and Robson 2017), and described as one of the most challenging clinical encounters in contemporary Western societies (Rief and Broadbent 2007). In consultation rooms, MUPSpatients experience great dissatisfaction (Lian and Robson 2017; Rosendal et al. 2013; Taylor et al. 2012; Weiland et al. 2012) and poor clinical outcomes (Rosendal et al., 2013; Weiland et al. 2012). Health professionals report feeling helpless, inadequate and pressurised (Anderson et al. 2012; Dowrick et al. 2008; Salmon et al. 2005). Interactional difficulties might be related to the ways in which doctors and patients experience, tolerate and act upon the uncertainty they have to deal with. Subjective perceptions of uncertainty might affect how both parties seek and exchange information, cope with uncertainty, and engage in shared decision-making (Hillen et al. 2017). In order to improve doctor-patient partnerships within the field of the medically unexplained, it might be useful to understand how doctors and patients collaborate to manage a mutual challenge: their inability to abolish medical uncertainty.

The aim of this paper is to study the ways in which interactional conflicts are sustained in a territory of medical uncertainty. Through a sociological analysis of qualitative experiential data provided in writing by patients living with long-term illnesses, we explore the interactional dynamics of clinical encounters related to medically unexplained physical symptoms. Our data are limited to two subgroups of MUPS-patients: medically unexplained long-term fatigue (often named Myalgic Encephalomyelitis-ME, or Chronic Fatigue SyndromeCFS), and medically unexplained seizures (commonly labelled psychogenic nonepileptic seizures-PNES, or non-epileptic attack disorder-NEAD). Both are medically unexplained contested chronic conditions with a wide range of severe debilitating symptoms. A vast majority of those who receive a diagnosis of medically unexplained fatigue or seizures (about three out of four) are women (Faro et al. 2016; McKenzie et al. 2010). While analysing the data, we asked: how do patients expect health professionals to respond to medical uncertainty; how are their expectations met, and in what ways are their expectations and experiences socially constructed and structurally conditioned? After classifying, presenting and discussing five main expectations, we explore their cultural embeddedness by situating patient experiences in their sociocultural terrain. Linking expectations and experiences of patients to underlying culturally contingent factors provide an alternative to the dominating individualistic perspective. Changing the "frame" within which we understand medically unexplained illnesses towards a cultural perspective might serve to unveil sources of persistent interactional difficulties between doctors and patients in territories of medical uncertainty. 


\section{Design and data analysis}

This study builds on qualitative data from two mixed-methods studies originating in Norway and England (one subset of data from each study). The data was collected through email surveys containing questions about how study participants experienced health care services received in relation to their ME/NES, performed to explore patient experiences among people living with medically unexplained conditions. This paper builds on text from their responses to open-ended questions in these surveys.

\section{Data and participants}

Dataset 1 consists of 256 participants. The data were generated from an open-ended question included in an email survey conducted in Norway in April-May 2013. Participants responded through an online system administered by a national centre for research data. Prior to distribution, the questionnaire was systematically tested and piloted among 143 people belonging to the targeted group. Invitations to participate were distributed to 811 members of the Norwegian ME-Association with known email addresses (about $40 \%$ of all members). Members were asked to refrain from participating if they were below the age of 16, or did not suffer from the condition themselves (health professionals, parents, others). Of the 488 people (60\% of those approached) who submitted a return, we excluded 22 who did not give information about their age, gender or diagnosis. Of the remaining 466, 256 participants (55\%) wrote free-text comments to the open-ended question: "Is there something that you want to tell us that you have not already done so far in this survey?". Ninety percent of respondents are women. Together, free-text comments amounted to 26,500 words (on average, 100 words per respondent). After removing identifying information, texts were translated to English by a professional translator. Translated texts were checked for consistency and accuracy by the first (native Norwegian) author. The study was granted ethical approval by the Norwegian Data Protection Official (id. 31784). Statistical data from sub-sets of female participants have been published (Lian and Hansen 2015; Hansen and Lian 2016a, b). Among other things, the statistical analysis revealed a strong association between positive patient experiences and congruence in doctors and patients views on the illness (Lian and Hansen 2015).

Dataset 2 consists of 141 participants. It was generated through an in-depth webbased survey advertised to members of 20 online support groups (not disclosed for reasons of confidentiality) for people with PNES/NEAD, referred to hereafter as non-epileptic seizures (NES). The survey was piloted among 25 people with the condition. The inclusion criteria were that participants had received a diagnosis of NES, and were over 18 years of age. Data were collected July-October 2016. A total of 141 participants submitted complete responses. Using the number of surveys started (289) as a proxy denominator suggests a completion rate of $49 \%$. Six participants who reported a diagnosis other than NES and six Australian participants were excluded from the study, leaving 129 participants. Of these, $87 \%$ of are women. The 
respondents are located in the UK and Ireland (72) and USA and Canada (57). Participants responded to three separate open-ended questions, two mandatory (A\&B) and one optional (C). Participants were asked to "please think about the" (A) "single best" and (B) "single worst" "interaction you have had with a health professional about NES" and to "In your own words, please describe what you" (A) "liked" and (B) "disliked" "about the interaction with the health professional?" (C): "In relation to NES, is there anything else you would like to tell us about your experiences of interacting with health professionals?" (69 participants responded). Together, texts amounted to around 20,000 words (on average, approximately 150 words per respondent). The study was granted ethical approval by Nelson Mandela University Human Research Ethics Committee (id. H16-RTI-RCD-002).

\section{Data analysis}

The purpose of the data analysis was to seek an interpretative understanding of participant's stories (Weber 1922), which means that they "hold truth in the sense that they allow us to understand reality from the point of view of the person expressing these comments" (De Maio 2010, p. 150). In line with this perspective, we do not question whether expressed experiences are right or wrong, good or bad.

Texts were analysed in collaboration between both authors using an inductive qualitative thematic approach (Braun and Clarke 2006), inspired by a grounded theory strategy (Charmaz 2014; Glaser and Strauss 1967) and a constant comparative method (Glaser and Strauss 1967). Themes for the analysis were drawn inductively from each of the two datasets by identifying expectations stated directly or indirectly through descriptions of both met and unmet expectations. In stage one of the analysis, we identified as many themes as possible that emerged across each dataset (no themes were defined in advance). In stage two, we compared the results of both datasets, and settled on a list of five themes that appeared particularly important for both groups. We then classified meaning units related to the five themes, consistently for all texts, while continuously discussing and revising our interpretations. Using our data-driven codes, we then analysed the data systematically, first individually and then both of us together. While searching for both manifest and latent meaning of the texts, we used the interrogative pronouns "what", "how", "who" and "why". De-condensation of key concepts used in the texts, such as "being understood" and "being believed", was a vital part of this process. To ensure trustworthiness and theoretical saturation, we formulated and reformulated competing interpretations several times through an exploratory and case-led approach, and we rechecked our reading several times.

\section{Results}

After inductively developing themes emerging throughout both datasets, five main themes became particularly apparent. We start each theme by quoting expectations stated either explicitly or indirectly. 


\section{Theme 1: Acknowledging the lack of medical knowledge}

Although ME and NES are given medical names (diagnoses), these conditions are neither medically explained nor substantiated by positive biomedical markers. Participants acknowledge the lack of medical knowledge about their illness, and they expect health professionals to do the same:

... I can't expect my GP to have this [specialist] knowledge, but I can expect my GP to listen to what I have to say and respect me a person (ME7302).

My primary care physician. She has always been very understanding and extremely helpful even when neither of us knows what we are looking for (NES42582634).

I have been taken seriously even though they [GPs] have had no understanding of my ME illness (ME7299).

According to both groups, acknowledging the lack of medical knowledge demands open-mindedness and a willingness to learn more:

I am actually very satisfied with my GP; she knows little about the illness but has shown an outstanding willingness to learn more (ME7469).

The neurologist told me he didn't know a lot about PNES but was very curious to learn (NES41429984).

Experiences of the opposite are more common in both groups:

...many [doctors] show disdain for patients when they themselves are uncertain and find themselves in unknown territory. Why can't doctors simply admit that they know little about this - instead of humiliating and mocking the patient when they are uncertain (ME7479).

Health professional are often evasive when answering questions, and there are subjects they seem keen to avoid (e.g. whether or not there is a psychogenic basis). They seem uncomfortable discussing the condition due to a lack of understanding (NES40933009).

I miss professional curiosity (ME7193).

Most of all, they dislike doctors who use unsubstantiated psychological theories:

... my symptoms were wrongly assumed to be mental (ME7802).

It is all too easy to blame psychological factors. It is often easy to do that when there is a dearth of medical knowledge and explanatory models (ME7202).

Health personnel in general all too easily utter the sentence: 'It is probably something mental' when there is something they can't explain (ME7471).

It is very rare to find anyone in the medical profession that has any idea what NEAD is, therefore I am now just treated as someone with psychiatric illness (NES41438757). 
Both groups appreciate professionals who show, not only an open mind, but also demonstrate a willingness to investigate their symptoms and discuss them with the patient, but that is often not the case:

I found that my GP was so determined that the illness was mentally conditioned that she didn't feel she needed to investigate any further (ME7460).

I do not get tests run, I do not typically get evaluated, and am usually immediately discharged without the doctor even checking for injuries (NES41489271).

\section{Theme 2: Being "believed" they are not "faking"}

Despite uncertainties, participants expect health professionals to accept their condition as "real". Contrary to this expectation, they describe health professionals who confuse the lack of knowledge and technological proofs of disease (bio-markers) with non-existing illness:

If neurologists don't see it in a scan it doesn't exist (NES44796812).

I was told by a specialist I was referred to that now I had been examined "from head to arse", so now I finally had to understand that there was nothing [physically] wrong with me (ME7090).

The way he [the neurologist] looked at me like I was crazy [...] basically telling me it was all in my head (NES44796812).

He [GP] yelled at me that this was a fake disease (ME7472).

[Neurologist] told me that there is nothing [physically] wrong with me (NES40949276).

One NES patient explained how seeing her seizures with their own eyes could be a 'proof-replacement':

I ended up having a seizure right in front of her [the GP] and she just completely accepted it. It was a nice change for once to have a doctor on my side (NES40958621).

Both groups expect caregivers to acknowledge their experiences and accept their condition as real, and treat this as fact. Having their experiences taken seriously and being reassured that they have a "real" illness and not an imaginary "fake" condition existing merely "in their head" is absolutely vital:

Fortunately I have found a GP who at least shows an understanding of the fact that I am ill (ME7310).

After many years of battling with my old GP, today I have a GP who believes me (ME7459).

She [neurologist] reassured me that these types of seizures are in no way fake (NES43726369). 
My GP sat down and explained this to me and made sure I understood it is not all in my head (NES40947994).

Experiences of the opposite are more common among participants in both groups:

.... it is difficult to find a GP .... who wants to relate to ME as an illness (ME7461).

.... a psychiatrist .... said straight out that ME didn't exist (ME7469).

He [GP] was so rude tried implying it was all in my head (NES40142452).

He [ER doctor] kept telling me I was faking and there is nothing wrong with me (NES41581556).

I haven't met a single one who believes this is an illness (NES41163538).

\section{Theme 3: Not being blamed}

Participants appreciate being reassured that their illness is involuntary and not something they consciously 'perform' or control, and therefore they should not be blamed for it. NES patients express this need with reference to positive experiences:

[Neurologist].... the first thing out of his mouth was that "it was not my fault." I needed to hear that! (NES43714636).

NES was explained to me and what was happening was not intentional and I didn't have control over it (NES40951076).

For most participants in both groups, however, these expectations are not met:

.... [Neurologist] told me it was all in my head and of my own doing .... said that because there were no abnormalities in my brain waves that it could be nothing else but voluntary (NES43714636).

In the end I collapsed from going for walks and was told [by GP] that 'since I didn't want to go for walks and take tablets, I could just go home. It was my own fault that I was ill!' (ME7210).

... brushed aside by my GP who told me it was attributable to unhappiness (so the cause was mental) (ME7803).

.... they put pressure on patients to 'pull themselves together' (ME7066).

The blaming often relates to insulting accusations of being stressed, lazy, attentionseeking or even hypochondriacs:

To be treated so badly, and also disbelieved and told that one is lazy, has an eating disorder, that it is a matter of willpower etc. when one is seriously ill, is a terrible additional strain (ME7066).

I was pretty much told [by hospital staff] that my condition didn't really exist and that I was just hysterical and an attention seeker (NES41116112). 
All around, .... [health professionals] treat PNES as if it were an imaginary friend. Fake, irrational, and made up to seek attention (NES42477133).

The Swedish doctor [rheumatologist]... concluded that I was a hypochondriac, diagnosis neurasthenia" (ME7462).

Some participants in the ME-group link the blaming to prejudice attitudes against women, or relate disbelief to male health professionals:

I have had doctors at the hospital who laugh at me. They ask if I am one of those stressed housewife types before I have even opened my mouth (ME7202).

A lot of focus on the 'good girl syndrome' (ME7321).

He [GP] knows nothing about women and nothing about patients and patient reactions (ME 7670).

The doctor [GP] I had after that was a man, and didn't take me so seriously (ME7440).

The participants link disbelief and blame to psychogenic explanations of their ailment:

They [neurologists] made me feel like it was my fault. That I somehow overstressed myself to the point that I couldn't just handle it. So my body was reacting with these "fake" pseudoseizures. In other words it was all in my head and could be controlled if [I] would just adjust the stress in my life (NES43726369).

\section{Theme 4: Demonstrate compassion, understanding and respect}

Both groups expect health professionals to listen to them and demonstrate understanding, compassion and respect:

They [neurologists] showed compassion and understanding (NES42581346).

My doctor [GP] since 2007 until the present has much more empathy and an open attitude and most important of all, she listens to what I say (ME7362).

Both groups primarily experience unmet expectations in this area:

It is upsetting how little knowledge the health services have about ME and how rude they are; they have no knowledge of the illness and don't listen to the patient at all (ME7562).

So frustrating as they [hospital staff] had no compassion or understanding and made me feel even worse about my condition (NES41575187).

Both groups express expectations of being treated as a (whole) human being:

For me, this illness is physical but of course it affects me mentally, I am after all a whole human being (ME7308). 
[Nurse] treated me like a human and that I was a genuine person.... (pretty unusual) (NES40933824).

This expectation is often not met:

They [specialists] don't take the tests I want, they don't believe me, they look for other things that are wrong, they overlook the problem, refuse to see the whole person, focus only on their specialism (ME7619).

\section{Theme 5: Share authority on decisions}

Both groups expect decisions to be made in a collaborative partnership with health professionals:

[GP] understands my needs, and supports me in what I want to do about my own health. I therefore think that my GP and I are a good team (ME7310).

She [GP] worked with me .... (NES42353316).

He [GP] was interested in listening to what I wanted (NES41610875).

.... We $[\mathrm{GP}]$.... discussed treatment options. I felt like I had been included in the decision-making and been given some control over my condition (NES44584667).

The medical profession must cooperate with those who know and have dealt with this for many years (ME7231).

Both groups express disappointment in relation to co-determination:

doctors give us far too little right of co-determination with regard to our own health; as long as there is no treatment, the goal is to alleviate the symptoms, and doctors must become better at listening to what the patient thinks works best for them (ME7203).

Today they are completely helpless when they encounter us, and therefore they react by distancing themselves, both from us as patients and from our illness (ME7057).

I often have a feeling that most doctors are not on the side of ME patients, on the contrary they are an opponent (ME7202).

[Neurologist]... threatened to not care for me if I disagreed with her diagnosis and treatment plan (NES40947994).

Both groups describe incidents indicating a total lack of partnership:

One literally yelled at me and physically threw me out of his office after throwing something at me from his desk (ME7066).

A general physician (local hospital), I was told, "never to go back there" (NES42227637). 
'I don't think they'll find anything,' he said. 'You don't need to come here anymore' (ME7202).

.... in a letter to my GP he wrote that under no circumstances must I be referred back to him (ME7462).

[Nurse to patient].... "get up you are wasting my time, why do I have to put up with patients like you" (NES4862903).

.... their main concern is to get rid of you (ME7625).

\section{Summary of results}

Through positive and negative descriptions, our study participants articulatedirectly or indirectly - the same basic expectations and experiences. Regarding expectations, the core message that runs through both datasets is that caregivers ought to

1. acknowledge the lack of medical knowledge; be frank, open and curious about medical uncertainties, and approach their condition with an open mind and a willingness to learn more (and above all, do not profess knowing what is not known);

2. believe patient experiences and the reality of their illness;

3. avoid moral blaming of patients;

4. treat patients with human dignity by demonstrating confidence, trust, respect, compassion, sympathy and understanding; and

5. share decision-making authority with patients in a collaborative partnership.

While positive experiences are expressed, the data are dominated by disappointment and dissatisfaction related to unmet expectations in all five themes. Participants' strongest aversion is against health professionals who claim to know what is not known by proclaiming psychogenic explanations of their illness, or characterise it as fake or non-existent. This might be the main source of negative experiences. Our participants interpret psychogenic explanations as indirect accusations that their symptoms are their own fault, and existing only "in their heads".

The only divergent pattern we found between the stories of the two groups is related to acknowledgement of their illness. Unlike ME-patients, some NES patients describe explicitly expressed acknowledgement from health professionals about their illness being "real" and not merely "faked". This is most likely related to differences between their symptoms: seizures are more acute, more specific, more directly observable from outside and often visibly fierce. Apart from that, the stories of both groups of participants are surprisingly similar. 


\section{The social and structural embeddedness of medically unexplained conditions: a theoretical analysis}

The consistency of our participants' stories indicates that their experiences ought to be interpreted as something more than individual assessments. Our assumption here is that they are consistent because patients' individual expectations and experiences are culture-bearing interpretations shaped by culturally available norms and values, and therefore socially conditioned and constructed. Contextualising their individual experiences by locating them in a specific time and place might reveal their social and structural embeddedness. Apart from being individual experiences, we analyse the stories of our participants as expressions of the contours of the modern biomedical paradigm, the changing roles of patients and health professionals, and the culturally and historically contingent norms and values of Western industrialised societies.

\section{Cultural norms and values}

The stories of our ME-participants mirror cultural views on tiredness in our culture. Our cultural norms define tiredness as a sign of weakness; the norm is to be energetic, tough, strong and endurable (Widerberg 2005). Cultural norms also prescribe how we ought to handle it: the (stereotyped) masculine response; pull yourself together and "handle it like a man", is the most culturally acceptable way (Widerberg 2005). Inability to cope with life events is also a core element in medical perceptions of non-epileptic seizures, and the gender dimension is visible there too: female preponderance is explained by the social acceptability of overt emotional expression in women (Reuber and Elger 2003).

This is just one example of how the stories of our participants reflect the hallmark of modernity in Western societies, most importantly the individualistic notion of disembedded individuals acting on the basis of free will. The individualistic 'triumph of the will' (Brown and Baker 2012, p. 32) has substituted societal factors with individual choices (unhealthy lifestyles) in medical explanations of diseases. The behavioural argument constitutes the core of a modern biomedical system in which individual freedom and responsibility are emphasised (Porter 2006). Within this perspective, allegedly susceptible vulnerable women who do not manage to live up to the ideals of culturally legitimate ways to handle tiredness and harsh life events risk social exclusion and stigmatisation from the successful majority.

Gendered or not, our participants describe how they experience being subjected to a stigmatising discourse with negative labelling and discrediting accusations (directed towards what they do and who they are) that leads to humiliation, shame, blame, stigma and social exclusion (Goffman 1963; Becker 1963). Because of the absence of medical acknowledgement, our participants experience what Freidson (1970) classifies as an illegitimate stigmatised illness. Being in this sick-role grants few, if any, privileges (ibid.). The psychiatric labels participants perceive to be awarded provide a stamp on behaviour that is regarded as socially unacceptable, and therefore often accompanied by social exclusion (Scheff 1974). 


\section{Modern biomedical perspectives}

Experiences of stigma expressed by our participants are also strongly linked to the modern biomedical paradigm, including the growing emphasis on evidence-based medicine. Within the biomedical paradigm, somatic symptoms without detectable organic pathology become unintelligible, incomprehensible, unexplainable and untreatable. It even becomes questionable whether their problems exist or not, because the criteria for existence-in an ontological sense-is what you can visually observe by the use technological tests. Among doctors, it is not uncommon to deny the very existence of MUPS-conditions (Anderson et al. 2012) or to explain them as psychogenic. In a culture where psyche and soma are separated and hierarchically structured (with the latter perceived as less real), and mental problems seen as signs of weakness, hence, self-inflicted (Jutel 2011), these medical perceptions easily lead to resentment from those who receive these labels.

Moreover, patients find it problematic when expert jurisdiction is claimed without the knowledge it ought to rely on, and knowledge is replaced with normative judgements. For doctors, scepticism towards MUPS, including the belief that it does not exist, reflects their professional biomedical view of the condition as such. Within a biomedical frame of reference, this is a value-neutral scientific statement (illness does not exist until bio-medically accounted for). Seen from an experiential perspective, patients easily perceive such statements not as value-neutral, but as personal, normative and judgmental. Based on cultural views of mental conditions, patients experience being held accountable for their illness and pressurised to pull themselves together, as if it was a "matter of willpower". If they refuse to comply with treatments, do not get better, or their condition deteriorates, they perceive it is they — not the treatment or clinician — who are held responsible (" "told ... it was my own fault that I was ill!'”). Consequently—whether intended or unintended-doctors transfer their scepticism towards the disease as a biomedical entity to patients' illness experiences. Without distinguishing clearly between bio-medically defined disease and experientially defined illness, interactional conflicts in territories of medical uncertainty can neither be understood nor resolved.

\section{New roles for doctors and patients}

The strong discontent expressed in the stories of our participants must be seen in relation to on-going political efforts to move towards a less authoritative professional role vis-á-vis the patient, and develop patient-centred care (Sacristán 2013). Patient-centred care means incorporating the experiential perspective of patients, and respecting patient autonomy to ensure that clinical decisions are made in concordance with patients' wishes and needs on the basis of shared decision-making (Hurtado et al. 2001). Recognising and respecting patients as persons with their own goals, expectations and needs is fundamental for this idea of clinical practice, which is rooted in a philosophy of people as purposeful, thinking, feeling, emotional, reflective and relational beings who are responsive to meaning (Cassell 2010). Delivering evidence-based medical services of high quality, consistent with 
the patient's medical needs and in a way that fosters a doctor-patient partnership of equals and shared decision-making, is qualities strived for in all Western health care systems. These ideological trends are absorbed by our participants, but old power structures deeply ingrained in the medical system might restrict ideals of patient autonomy to mature, and thereby obstruct the process of change.

\section{Strengths and limitations}

Data were collected independently of service providers, and the identity of our participants was never revealed to the researchers. This allowed respondents to describe their experiences without fearing negative consequences from healthcare providers. Giving them open-ended questions and allowing them to describe their experiences in writing - with minimal researcher influence, no time-pressure and no wordlimit - granted them full freedom to reflect on whatever they found most important. Despite this freedom, participants distributed across Western nations told us remarkably similar stories, which we interpret as a validation of the openness of questions raised: it reveals that despite place of residence, MUPS-patients encounter common challenges that stem from having an illness that is impossible to "validate" through medical technology and knowledge. Using written texts instead of structured oral interviews, which has been the most common method so far, makes our study an original supplement to previous research, even in an international context.

Because of recruitment methods (based on membership of patient organisations or online support groups), the experiences of our participants might not be the same as other people with these conditions. A possible bias is that those who chose to participate in our study have more negative experiences than those who did not. We therefore do not claim that the views of our participants are representative for all patients with ME and NES, but they are useful in terms of exploring cultural and contextual factors contributing to interactional challenges. Our data might also be biased towards higher functioning patients (which is to be expected, as those most seriously affected were probably unable to participate). Also, men are underrepresented in both patient groups. While interpreting the data, these possible biases have to be taken into account. We must also bear in mind that the stories of our participants are retrospective (they recall experiences from consultations that happened some time ago), and therefore change as time goes by. Another weakness of our data is that the two patient groups were not asked identical questions. This renders a systematic comparative analysis of the two participant groups impossible.

\section{Conclusion}

Directly and indirectly, participants in this study express clear expectations towards caregivers: through sympathy, empathy and compassion, health professionals ought to demonstrate that they acknowledge patient experiences in a non-judgmental way, and act upon it through shared decision-making. In an insightful manner, the 
participants describe unmet expectations in relation to all these aspects. One participant summarises her experiences in a way that illuminates a core message given in our data: "All interactions have been negative with blaming, shaming, humiliation, and emotional pain (NES43349947)". Most participants describe tension-filled encounters dominated by interactional conflicts containing unresolved credibility and knowledge claims, demeaning behaviour and humiliating accusations.

In our culture, we often explain the ways in which human beings think, act and feel by employing a psychological perspective of individual personality and personal motives. Interactional difficulties discussed in this paper make no exception. "Why are ME-patients so angry?" a doctor asks (Johnson 2015). Similarly, one of our participants asks: why can't doctors stop "humiliating and mocking the patient? (ME7479)". A major problem with this perspective is that is misses the inherently sociocultural dimension of our thoughts, actions and feelings. Through social interaction, cultural norms and values become our internalised norms and values. By exploring how individual experiences convey the sociocultural context in which they are nurtured, we have tried to move the frame of reference to a cultural level and offer a contextual perspective on the interactional dynamics of clinical consultations fused by medical uncertainty. This perspective also entails explanatory power: it serves to explain why expectations and experiences expressed by our study participants are so coherent.

In this paper, we interpret the stories of our participants as expressions of an experienced systematic disrespect that transpires from the naturalistic empiricism of the modern western scientific biomedical paradigm, and from cultural norms and values of its surrounding society. The interactional dynamics they describe exhibit structurally conditioned forces inherent in the medical system, in the consultation room and in the minds of those who interact there. Although people handle the situation differently, structural forces influence the ways in which actors think and act. Acknowledging the structurally conditioned circumstances we act upon means accepting that experiences and expectations of all those who engage in the consultation room (patients as well as health professionals) are contingent upon a specific cultural context. Situating events within this context enable us to identify the underlying dynamics of structurally generated conflicts, and thereby prevent individual moral blame. Coupling agency and structure by exploring patient experiences in relation to medical and sociocultural systems reveals how the interactional dynamics of medical consultations in territories of medical uncertainty is socially constructed and structurally conditioned.

OpenAccess This article is distributed under the terms of the Creative Commons Attribution 4.0 International License (http://creativecommons.org/licenses/by/4.0/), which permits unrestricted use, distribution, and reproduction in any medium, provided you give appropriate credit to the original author(s) and the source, provide a link to the Creative Commons license, and indicate if changes were made. 


\section{References}

Anderson, V.R., L.A. Jason, L. Hlavaty, N. Porter, and J. Cudia. 2012. A review and meta-synthesis of qualitative studies on myalgic encephalomyelitis/chronic fatigue syndrome. Patient Education and Counseling 86 (2): 147-155.

Banks, J., and L. Prior. 2001. Doing things with illness. The micro politics of the CFS clinic. Social Science and Medicine 52, 1: 11-23.

Becker, H. 1963. Outsiders: Studies in the sociology of deviance. London: Macmillan.

Braun, V., and V. Clarke. 2006. Using thematic analysis in psychology. Qualitative Research in Psychology 3 (2): 77-101.

Brown, B., and S. Baker. 2012. Responsible citizens: Individuals, health and policy under neoliberalism. London: Anthem Press.

Busfield, J. 2017. The concept of medicalisation reassessed. Sociology of Health \& Illness 39 (5): $759-774$.

Cassell, E.J. 2010. The person in medicine. International Journal of Integrated Care 10 (5): e019.

Charmaz, K. 2014. Constructing grounded theory. Los Angeles: SAGE.

De Maio, F. 2010. Health \& social theory. Basingstoke: Palgrave Macmillan.

de Wolfe, P. 2009. ME: The rise and fall of media sensation. Medical Sociology Online 4 (1): 2-13.

Dowrick, C., L. Gask, J. Hughes, H. Charles-Jones, J.A. Hogg, and S. Peters. 2008. General practitioners' views on reattribution for patients with medically unexplained symptoms: A questionnaire and qualitative study. BMC Fam Pract 9: 46.

Faro, M., N. Saez-Francas, J. Castro-Marrero, L. Aliste, T. Fernandez de Sevilla, and J. Alegre. 2016. Gender differences in chronic fatigue syndrome. Reumatologia Clinica 12 (2): 72-77.

Freidson, E. 1970. The profession of medicine. A study of the sociology of applied knowledge. Chicago: University of Chicago Press.

Glaser, B.G., and A.L. Strauss. 1967. The discovery of grounded theory: Strategies for qualitative research. New York: de Gruyter.

Goffman, E. 1963. Stigma: Notes on the management of spoilt identity. New Jersey: Prentice Hall.

Hansen, A.H., and O.S. Lian. 2016a. Experiences of general practitioner continuity among women with chronic fatigue syndrome/myalgic encephalomyelitis: A cross-sectional study. BMC Health Services Research 16 (1): 650. https://doi.org/10.1186/s12913-016-1909-1.

Hansen, A.H., and O.S. Lian. 2016b. How do women with chronic fatigue syndrome/myalgic encephalomyelitis rate quality and coordination of healthcare services? A cross-sectional study. BMJ Open 6 (4): e010277. https://doi.org/10.1136/bmjopen-2015-010277.

Han, P.K.J., W.M.P. Klein, and N.K. Arora. 2011. Varieties of uncertainty in health care: A conceptual taxonomy. Medical Decision Making 31 (6): 828-838.

Hillen, M.A., C.M. Gutheil, T.D. Strout, E.M.A. Smets, and P.K.J. Han. 2017. Tolerance of uncertainty: Conceptual analysis, integrative model, and implications for healthcare. Social Science and Medicine 180: 62-75.

Hurtado, M., E. Swift, and J. Corrigan. 2001. Institute of Medicine (US) Committee on the National Quality Report on Health Care Delivery. Washington, DC: Envisioning the National Health Care Quality Report.

Jewson, N.D. 2009. The disappearance of the sick-man from medical cosmology, 1770-1870. International Journal of Epidemiology 38: 622-633.

Johnson, G. 2015. Hvorfor er ME-pasienter så sinte? Dagens medisin 2: February.

Jutel, A.D. 2011. Putting a name to it. Diagnosises in contemporary society. Baltimore: Johns Hopkins University Press.

McKenzie, P., M. Oto, A. Russell, A. Pelosi, and R. Duncan. 2010. Early outcomes and predictors in 260 patients with psychogenic nonepileptic attacks. Neurology 74 (1): 64-69.

Lian, O.S., and H. Bondevik. 2015. Medical constructions of long-term exhaustion-past and present. Sociology of Health \& Illness 37 (6): 920-935. https://doi.org/10.1111/1467-9566.12249.

Lian, O.S., and A.H. Hansen. 2015. Factors facilitating patient satisfaction among women with medically unexplained long-term fatigue: A relational perspective. Health 20 (3): 308-326. https:// doi.org/10.1177/1363459315583158.

Lian, O.S., and S. Nettleton. 2015. 'United we stand'. Framing myalgic encephalomyelitis in a virtual symbolic community. Qualitative Health Research 25 (10): 1383-1394. https://doi. org/10.1177/1049732314562893. 
Lian, O.S., and C. Robson. 2017. It's incredible how much I've had to fight. Negotiating medical uncertainty in clinical encounters. International journal of qualitative studies on health and well-being 12 (1): 1392219.

Parsons, T. 1951. The social system. Glencoe: The Free Press.

Porter, D. 2006. How did social medicine evolve, and where is it heading? PLoS Medicine 3 (10): e399.

Reuber, M., and C.E. Elger. 2003. Psychogenic nonepileptic seizures: Review and update. Epilepsy \& Behavior 4 (3): 205-216.

Rief, W., and E. Broadbent. 2007. Explaining medically unexplained symptoms-models and mechanisms. Clinical Psychology Review 27 (7): 821-841.

Rosendal, M., A. Blankenstein, R. Morriss, P. Fink, M. Sharpe, and C. Burton. 2013. Enhanced care by generalists for functional somatic symptoms and disorders in primary care. Cochrane Database Systematic Review 18, 10: CD008142.

Sacristán, J.A. 2013. Patient-centered medicine and patient-oriented research: Improving health outcomes for individual patients. BMC Medical Informatics and Decision Making 13 (1): 1-8.

Salmon, P., A. Ring, C.F. Dowrick, and G.M. Humphris. 2005. What do general practice patients want when they present medically unexplained symptoms, and why do their doctors feel pressurized? Journal of Psychosomatic Research 59 (4): 255-260.

Scheff, T.J. 1974. The labelling theory of mental illness. American Sociological Review 39 (3): 444-452.

Smith-Rosenberg, C. 1986. Disorderly conduct: Visions of gender in victorian. Oxford: University of Oxford Press.

Taylor, R.E., T. Marshall, A. Mann, and D.P. Goldberg. 2012. Insecure attachment and frequent attendance in primary care: A longitudinal cohort study of medically unexplained symptom presentations in ten UK general practices. Psychological Medicine 42 (4): 855-864.

Toombs, S.K. 1992. The meaning of illness. A phenomenological account of the different perspectives of physician and patient. London: Kluwer Academic Publishers.

Weber, M. 1922. [1978]. Economy and society: An outline of interpretive sociology. Berkley, CA: University of California Press.

Weiland, A., R.E. Van de Kraats, A.H. Blankenstein, J.L.C.M. Van Saase, H.T. Van der Molen, W.M. Bramer, A.M. Van Dulmen, and L.R. Arends. 2012. Encounters between medical specialists and patients with medically unexplained physical symptoms; influences of communication on patient outcomes and use of health care: A literature overview. Perspectives on Medical Education 1 (4): 192-206.

Widerberg, K. 2005. Embodied gender talks: The gendered discourse of tiredness. In Gender, bodies and work, ed. D. Morgan, B. Brandth, and E. Kvande. Aldershot: Ashgate.

Wright, P., and A. Treacher. 1982. Introduction. In The problem of medical knowledge. Examining the social construction of medicine, ed. P. Wright and A. Treacher. Edinburgh: Edinburgh University Press. 